\title{
Dynamic Transport Study of the Plasmas with Transport Improvement in LHD and JT-60U
}

K. Ida et al. 
Topic : EX/P4-39

\title{
Dynamic Transport Study of the Plasmas with Transport Improvement in LHD and JT-60U
}

\author{
K.Ida 1), Y.Sakamoto 2), S.Inagaki 1), H.Takenaga 2), A. Isayama 2), R.Sakamoto 1), \\ K.Tanaka 1), S.Ide 2), T.Fujita 2), H.Funaba 1), S.Kubo 1), M.Yoshinuma 1), \\ T.Shimozuma 1), Y.Takeiri 1), K.Ikeda 1), C.Michael 1), T.Tokuzawa 1), LHD experimental \\ group and JT-60 team
}

1) National Institute for Fusion Science, Oroshi, Toki, 509-5292, Japan

2) Japan Atomic Energy Agency, Naka, Ibaraki-ken, 311-0193, Japan

e-mail contact of main author: ida@nifs.ac.jp

\begin{abstract}
A transport analysis during the transient phase of heating (a dynamic transport study) applied to the plasma with internal transport barriers (ITBs) in the Large Helical Device (LHD) heliotron and JT-60U tokamak is described. In the dynamic transport study 1) a slow transition between two transport branches is observed, 2) the time of the transition from the L-mode plasma to the ITB plasma is clearly determined by the onset of the flattening of the temperature profile in the core region and 3) a spontaneous phase transition from a weak, wide ITB to a strong, narrow ITB and its back-transition are observed. The flattening of the core region of the ITB transition and the back-transition between a wide ITB and a narrow ITB suggest the strong interaction of turbulent transport in space, where turbulence suppression at certain locations in the plasma causes the enhancement of turbulence and thermal diffusivity nearby.
\end{abstract}

\section{Introduction}

The concept of a profile transition has been employed in order to understand the formation and dynamics of a self-sustained radial profile in inhomogeneous plasmas[1].The transitions in the profiles in H-mode plasmas are caused by the S-curve property (cusp-type catastrophe) in the gradient-flux relation, showing a feature analogous to the first-order phase transition[2]). The slow transition to the improved transport mode[3], which is often observed in other types of improved modes or internal transport barrier (ITB) plasmas[4], is categorized as a type of second-order transition in contrast to a first-order transition such as the $\mathrm{L} / \mathrm{H}$ transition[5-7]. In helical plasma, associated with the transition from ion-root to electron-root, an electron internal transport barrier (ITB) appears, when the heating power of the ECH exceeds a threshold power[8-12].

In this type of transition, it has been difficult to determine the time of the transition, because the temperature at the ITB region increases continuously and there is no clear jump observed. A dynamic transport study, where the time evolution of the electron temperature $\left(T_{e}\right)$ gradient and its relation to the heat flux normalized by density $Q_{e} / n_{e}$ are analyzed in time, is found to be a useful tool for defining the time for transition and for studying the mechanism of ITB formation. Flattening of the electron temperature profile inside the strong ITB is observed in JT-60U[13], while there is no significant flattening of the electron temperature profile observed in LHD[14]. Although the general differences in the electron temperature profiles inside the ITB between LHD and JT-60U imply the importance of magnetic field structure in determining the electron temperature profile, it is also observed that the radial profile of the temperature varies in time even without a change of magnetic structure. This fact suggests that there are multiple states within ITB and plasma can make transition between ITB states[15,16]. 
In this paper, dynamic transport studies applied to the plasmas with pellet improved mode in the Large Helical Device (LHD) heliotron and to plasmas with an ITB in the JT-60U tokamak are described. The transition time from an L-mode plasma to an ITB plasma (ITB formation timing) and the transition between multiple ITB states (one is weak ITB with a parabolic-shaped profile and the others are wide or narrow ITB with a box-type profile[15]) observed in LHD and JT-60U are discussed.

\section{Dynamic Transport Analysis for the Discharhe with Pellet Injection in LHD}

In order study the dynamic transport the normalized heat flux is scanned by changing the electron density using repetitive pellets in the $\operatorname{LHD}(\mathrm{R}=3.5 \mathrm{~m}, \mathrm{a}=0.6 \mathrm{~m} \mathrm{~B}=2.8 \mathrm{~T})$,. The size of the repetitive pellets is $2.5 \mathrm{~mm}$ diameter and the volume averaged density increases by $1.6 \times 10^{19} \mathrm{~m}^{-3}$ with one pellet. The speed of the pellet is $450-500 \mathrm{~m} / \mathrm{s}$ and the pellets penetrate as far as from half of the plasma.

Figure 1(a) show the time evolution of the electron temperature gradients during the density decay phase after the pellet injection, where the normalized heat flux increases monotonically in time by a factor of two. There are clear phases characterized by the sign of the time derivative of the $T_{e}$ gradients as indicated by phases I, II, III, IV. Both $T_{e}$ and the $T_{e}$ gradient increase in time as the electron density decreases in phases II and IV, which is a normal characteristic of heat transport in plasma. However, in phases I and III, the $T_{e}$ gradients decrease even with the increase of $T_{e}$. The change in the time derivative of the $T_{e}$ gradient is abrupt (less than $0.01 \mathrm{sec}$ ) and its value is roughly constant in time for each phase as $-6.6 \mathrm{keV} / \mathrm{m} / \mathrm{s}$ (in phase I), $2.2 \mathrm{keV} / \mathrm{m} / \mathrm{s}$ (in phase II), $-2.7 \mathrm{keV} / \mathrm{m} / \mathrm{s}$ (in phase III) and 0.6 $\mathrm{keV} / \mathrm{m} / \mathrm{s}$ (in phase IV). This observation clearly demonstrates that the back-transition phase between the two meta-stable states is characterized by the change in the time derivative of the $\mathrm{T}_{\mathrm{e}}$ gradient.
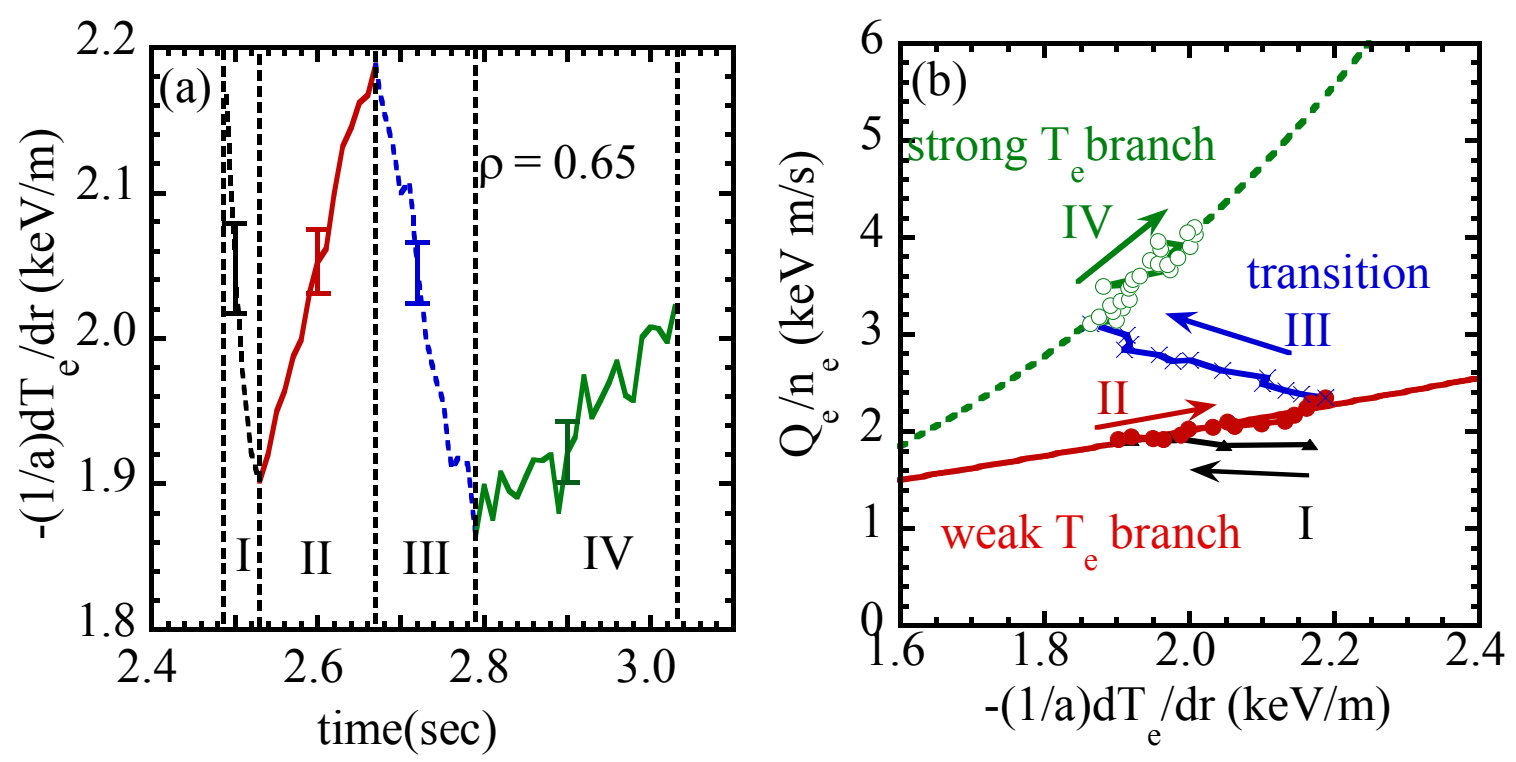

FIG.1. (a) Time evolution of the temperature gradient after pellet injection ( $t=2.48 \mathrm{sec})$ and (b) heat flux normalized by electron density as a function of electron temperature gradient at $\rho=0.65$ after pellet injections.

The change in transport is more clearly presented in the relation between the heat flux normalized by the electron density and the $\mathrm{T}_{\mathrm{e}}$ gradient as seen in Fig.1(b). There are two branches in the transport, one is characterized by a weak $T_{e}$ dependence (lower branch : phase II) and the other by a strong $T_{e}$ dependence (upper branch: phase IV). Phase III in Fig. 1 is the transition phase between the strong and weak $\mathrm{T}_{\mathrm{e}}$ dependence branches. During the 
back-transition phase, the heat flux normalized by the electron density has a negative dependence on the absolute value of the $T_{e}$ gradient as $d\left(Q_{e} / n_{e}\right) / d\left(-d T_{e} / d r\right)<0$ which is in contrast to the normally positive dependence of the normalized heat flux in the two transport branches.

In order to investigate quantitatively the dependence of the normalized heat flux on the $\mathrm{T}_{\mathrm{e}}$ and $\mathrm{T}_{\mathrm{e}}$ gradient dependence, the normalized heat flux is given by $\mathrm{Q}_{\mathrm{e}} / \mathrm{n}_{\mathrm{e}}=\mathrm{cT}_{\mathrm{e}}{ }^{\alpha}(\mathrm{d} \mathrm{T} \mathrm{e} / \mathrm{dr})^{\beta}$, where $\alpha$ and $\beta$ are the $T_{e}$ and $T_{e}$ gradient dependence parameters, respectively. The difference between the $Q_{e} / n_{e}$ measured and $Q_{e} / n_{e}$ calculated with the parameters $(\alpha, \beta)$ are investigated in a wide range of $Q_{e} / n_{e}$ for these two branches. The parameters $(\alpha, \beta)$ are evaluated by taking the set of $(\alpha, \beta)$ that gives a minimum value of the square of differences between the normalized heat flux measured and that calculated. The $T_{e}$ dependence parameter $\alpha$ is 0.44 for the weak $T_{e}$ dependence transport branch and 1.4 for the strong $T_{e}$ dependence transport branch, while the $T_{e}$ gradient dependence parameter, $\beta$ is close to unity ; 0.86 and 1.1 for the weak and the strong $\mathrm{T}_{\mathrm{e}}$ dependence branches, respectively.
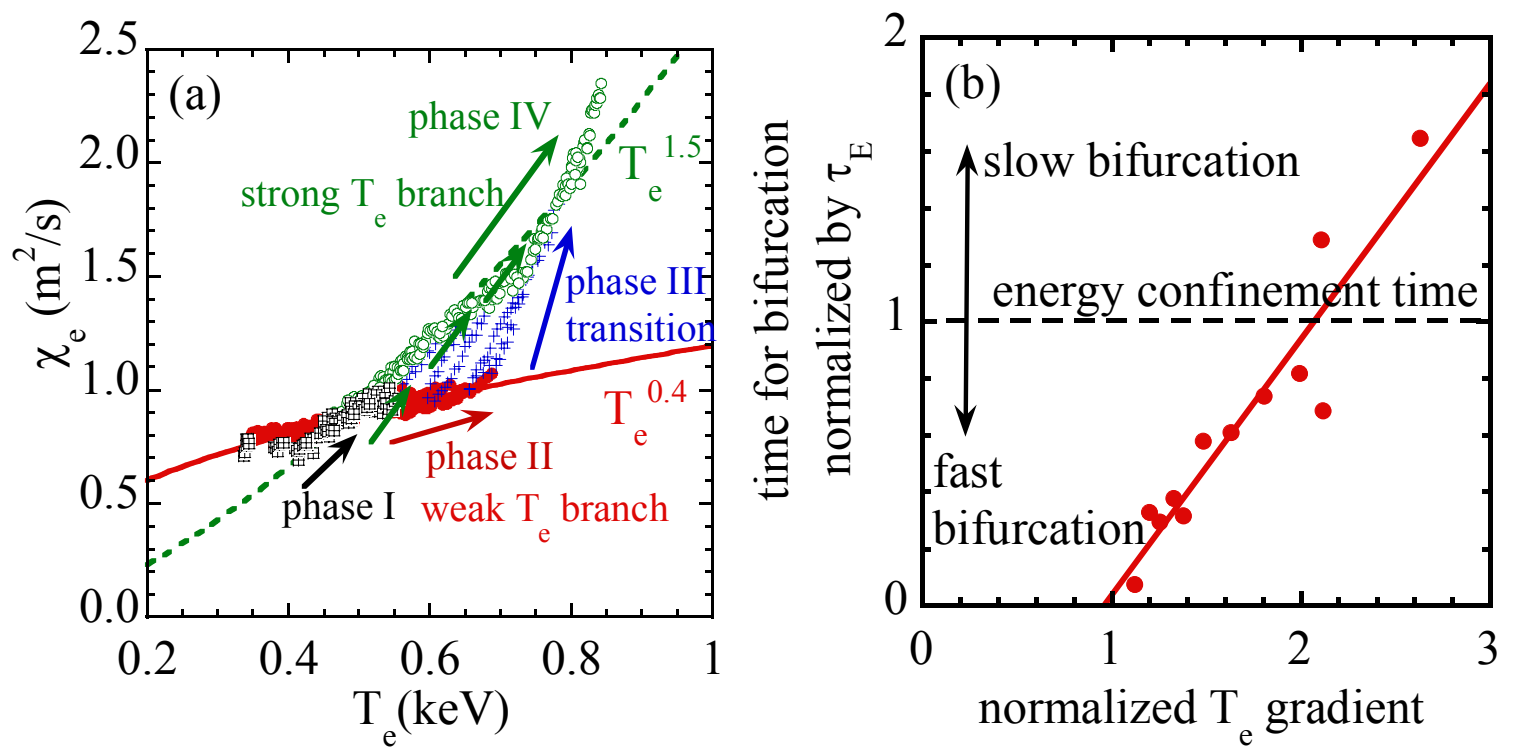

FIG.2 (a)Temperature dependence of thermal diffusivity in Phase I, II, III, IV. A slow transition from a weak to a strong $T_{e}$ dependence transport branch is observed. (b)Time for the transition (phase III) normalized by the energy confinement time as a function of the $T_{e}$ gradient normalized by the critical $T_{e}$ gradient, where two branches are merging.

The differences of transport between the two branches are characterized by the different temperature dependence not the different temperature gradient dependence. Furthermore the normalized heat flux is roughly proportional to the temperature gradient in this experiment, the difference between the two branches can be evaluated with a standard thermal diffusivity of $\chi_{\mathrm{e}}=\mathrm{Q}_{\mathrm{e}} / \mathrm{n}_{\mathrm{e}} /\left(\mathrm{dT}_{\mathrm{e}} / \mathrm{dr}\right)$ as seen in Fig2(a). There are two branches in the transport with different $\chi_{\mathrm{e}}$, one is characterized by a weak $\mathrm{T}_{\mathrm{e}}$ dependence (lower branch : phase II $\chi_{\mathrm{e}}=\mathrm{cT}_{\mathrm{e}}{ }^{0.4}$ ) and the other by a strong $\mathrm{T}_{\mathrm{e}}$ dependence (upper branch: phase IV $\chi_{\mathrm{e}}=\mathrm{cT}_{\mathrm{e}}{ }^{1.5}$ ). These two transport branches discussed above merge as $\mathrm{T}_{\mathrm{e}}$ and the $\mathrm{T}_{\mathrm{e}}$ gradient become small enough, which is defined as the critical $T_{e}$ gradient. Below the critical $T_{e}$ gradient, only the weak $T_{e}$ dependence branch, which becomes the upper branch in turn, is observed. In the discharges without the perturbations of pellet injection, the plasma remains in only the upper branches (the strong $T_{e}$ dependence branch above the critical $\mathrm{T}_{\mathrm{e}}$ gradient and the weak $\mathrm{T}_{\mathrm{e}}$ dependence branch below the critical $\mathrm{T}_{\mathrm{e}}$ gradient) and the heat transport changes its $\mathrm{T}_{\mathrm{e}}$ dependence at this critical $\mathrm{T}_{\mathrm{e}}$ [17]. The time for the back-transition depends on the $T_{e}$ gradient and can be as large as the energy confinement time. As shown in Fig.2(b), the time for the transition depends on the $T_{e}$ gradient and it increases linearly as the ratio of the $T_{e}$ gradient to the critical $T_{e}$ gradient 
increases. The time for the transition is $0.1-1.6$ times the energy confinement time. The time for the transition can be as large as the energy confinement time, which is in contrast to the fast transition as observed in the $\mathrm{L} / \mathrm{H}$ transition and electric pulsation where the bifurcation of the radial electric field plays a role.

The observation of two distinct turbulent states casts illumination on the understanding of turbulent transport. Because of the nonlinear coupling between different types of turbulence, slow transitions between two states with different dominant turbulence types are theoretically predicted[18]. The identification of the two transport branches in the LHD experiment provides a manifestation of these theoretical analyses and stimulates further theoretical study on nonlinear competition among the different types of turbulence.

\section{Dynamic Transport Analysis in the ITB Plasma in JT-60U}

In order study the dynamic transport the normalized heat flux is scanned by adding the electron cyclotron heating $(\mathrm{ECH})$ to the NBI heated plasmas in the JT-60U $(\mathrm{R}=3.4 \mathrm{~m}, \mathrm{a}=0.9 \mathrm{~m}$, $\mathrm{B}=3.6 \mathrm{~T}, \mathrm{I}=1 \mathrm{MA})$. Figure 3 shows the relation between the electron temperature gradient and the heat flux normalized by the electron density after the ECH pulse is turned on in the plasma with negative magnetic shear in JT-60U. As seen in Fig3(a), the temperature gradient increases inside the ITB $(\rho=0.35)$. However, the temperature gradient near the boundary of the ITB ( $\rho=0.25$ shoulder in the $T_{e}$ profile) starts to decreases after the transition to the ITB, although the heat flux itself continuously increases. In contrast, the temperature gradients at $\rho$ $=0.35$ and $\rho=0.25$ increase as the normalized heat flux is increased, when there is no ITB
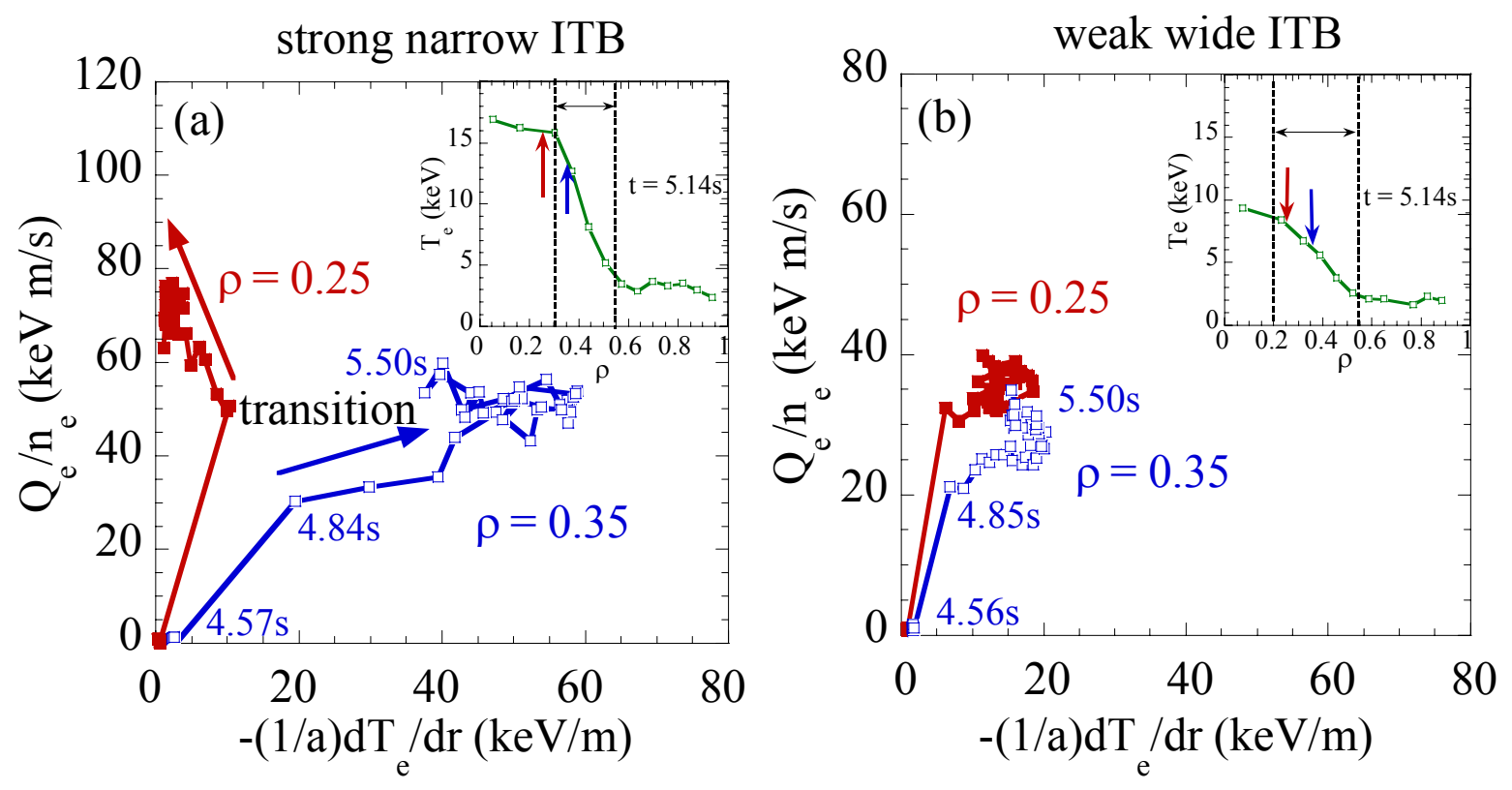

FIG.3. Heat flux normalized by electron density as a function of electron temperature gradient at electron internal transport barrier (ITB) region $(\rho=0.35)$ and the boundary of ITB region ( $\rho=$ 0.25 ) in the plasma with ECH (3MW in (a) )in negative magnetic shear configuration. There is no ITB formation when the ECH power is low (1.5MW in (b)) Radial profiles of electron temperature after the formation of ITB are also plotted. Dashed lines represent the ITB region while the arrows indicate the location where the dynamic transport analysis is applied for $t=$ $4.57-5.5 \mathrm{sec}$.

formation (Fig2(b)). The temperature gradient at $\rho=0.25$ recovers after the back-transition of ITB associated with the step down of ECH power. The observation of the negative dependence of the heat flux $\mathrm{d}\left(\mathrm{Q}_{\mathrm{e}} / \mathrm{n}_{\mathrm{e}}\right) / \mathrm{d}\left(-\mathrm{dT} \mathrm{T}_{\mathrm{e}} / \mathrm{dr}\right)<0$ near the ITB boundary suggests that the degradation of transport occurs associated with the formation of an ITB. This degradation is considered to be due to the enhancement of turbulence at the boundary between the ITB 
region and the L-mode region, where turbulence is excited by the nearby large temperature gradient.

\section{Time of the Transition from the L-mode Plasma to the ITB Plasma}

Figure 4 shows the time evolution of the $\mathrm{T}_{\mathrm{e}}$ gradients during the formation of electron ITBs in LHD and JT-60U after the ECH pulse is turned on in the NBI heated plasmas with low density $\left(\mathrm{n}_{\mathrm{e}}=0.5 \times 10^{19} \mathrm{~m}^{-3}\right.$ in LHD and $1 \times 10^{19} \mathrm{~m}^{-3}$ in JT-60U). In LHD, the temperature gradient at $\rho=0.17$ and $\rho=0.3$ increases on a transport time scale $(\sim 35 \mathrm{~ms})$ after the ECH pulse is turned on. The temperature gradient keeps increasing at $\rho=0.3$, which becomes the high gradient ITB region, while the temperature gradient starts to decrease at $\rho=0.17$, which becomes the "shoulder" of the ITB. Similar behaviour is observed during the formation of the parabolic-shaped ITB in JT-60U with positive magnetic shear, where the central q value is low enough not to cause the degradation of the transport[19]. The time of the abrupt change in the sign of the time derivative of the temperature gradient $(1.335 \mathrm{sec}$ in LHD and $5.105 \mathrm{sec}$ in JT-60U) is considered to be the time of the ITB formation (the transition from the L-mode to the ITB plasmas). It should be noted that the flattening of the temperature profile in the core region is observed both in positive and negative magnetic shear configurations in JT-60U and in LHD plasmas, where the magnetic shear is always negative and unchanged.

These observations indicate that the decrease of the temperature gradient at the ITB shoulder is due to the development of the ITB itself not due to the change of the magnetic configuration. The abrupt decrease of temperature gradient inner region associated with the increase of temperature gradient at the ITB region are in contrast to the non-local phenomena where the temperature gradient starts to increase associated with the decrease of temperature gradient at outer region[20,21]. The decrease of temperature gradient inner region at the time of the transition is not due to the increase of temperature but due to the localization of improved transport associated with the formation of the ITB.
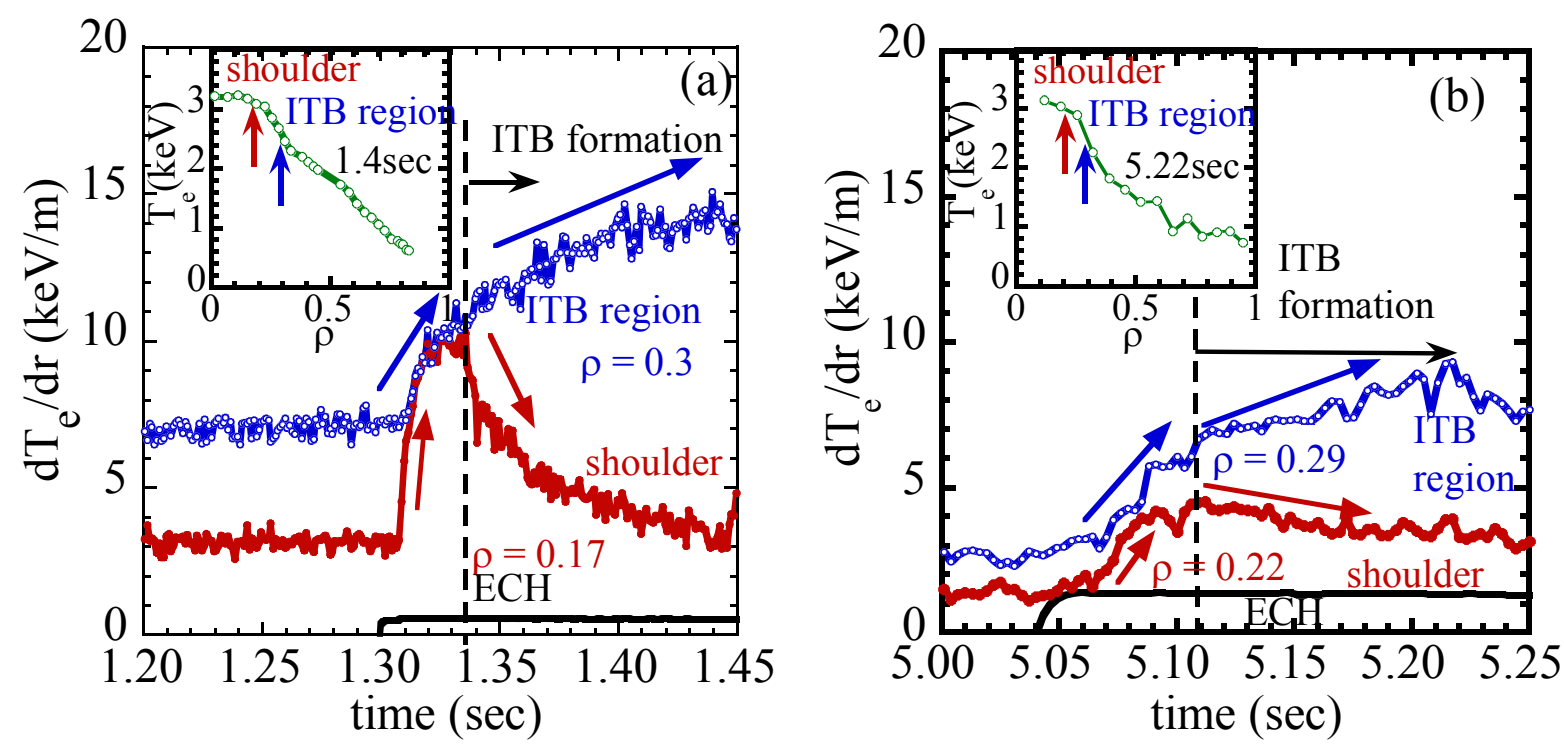

FIG.4.Time evolutions of the electron temperature gradients in the high gradient ITB region and at the shoulder during the formation of the ITBs in (a) LHD and (b) JT-60U with positive magnetic shear. An abrupt change in the sign of the time derivative of the temperature gradient at the "shoulder" shows there is a clear transition from the L-mode plasma to the ITB plasma and the time of the transition is indicated with dashed lines. Radial profiles of electron temperature after the formation of ITB are also plotted. Arrows indicate the location where the time evolutions of temperature gradient are plotted. 


\section{Transition between a weak wide ITB to a strong narrow ITB}

The flattening of the temperature profile in the core region causes a narrowing of the ITB region, where the temperature gradient is large, because the location of the foot point of the ITB is almost unchanged in this discharge. The narrowing of the ITB is more significant in the strong ITB such as the box-type ITB in JT-60U. In contrast, the radial profile of temperature becomes parabolic-shaped in the plasma with a weak, wide ITB. As seen in Fig.5, the spontaneous transition from a weak ITB to a strong, narrow ITB is observed during the phase of constant heating power both in LHD and JT-60U. In LHD, the decrease of the temperature gradient at $\rho=0.17$ is observed at $t=1.62 \mathrm{sec}$, which indicates the transition from a weak ITB $\left(\mathrm{R} / \mathrm{L}_{\mathrm{T}}=16\right)$ to a strong ITB $\left(\mathrm{R} / \mathrm{L}_{\mathrm{T}}=24\right)$. The abrupt increase of the temperature gradient at $\rho=0.32$ and the drop of the temperature gradient at $\rho=0.27$ at $t=1.8 \sec$ shows that the transition from a wide ITB to a narrow ITB. It should be noted that the time scale of the transition is $20 \mathrm{~ms}$, which is the half of the global energy confinement time of $43 \mathrm{~ms}$ and much shorter than the time scale of change in rotational transform and magnetic shear.

In JT-60U, the oscillation between the weak ITB $\left(\mathrm{R} / \mathrm{L}_{\mathrm{T}}=24\right)$ and the strong, narrow ITB $\left(\mathrm{R} / \mathrm{L}_{\mathrm{T}}=40\right)$ is observed. The growing of a strong ITB associated with the narrowing of the ITB region appears in the periods of $\mathrm{t}=5.04-5.16 \mathrm{sec}, \mathrm{t}=5.40-5.47 \mathrm{sec}$ and $\mathrm{t}=5.62-5.78$ $\mathrm{sec}$ and these strong, narrow ITBs are terminated and show back transitions. The temperature gradient at the foot point $(<5 \mathrm{keV} / \mathrm{m})$ is relatively constant in time during the oscillations. It is interesting that the strong, narrow ITB appears even during the decay of the central electron temperature after the ECH pulse is tuned off. The behaviour of the temperature gradient at the forward and backward transition of the strong, narrow ITB is consistent with that of ITB formation and also indicates that there are multi meta-stable states in the transport and the slow transition between these occurs on a transport time scale. The transition from the parabolic-shaped ITB to box-shaped ITB and second transition from the wide box-shaped ITB to narrow box-shaped ITB have been observed in the ion temperature profiles during the development of ITB[15], which also suggests the multiple state of ion transport. The observation of both the forward and backward transition of the electron transport in LHD and JT-60U strongly support the existence of multi meta-stable states of transport as a nature of transport and that the abrupt change of temperature gradient is not due to the change in magnetic shear of q profile.
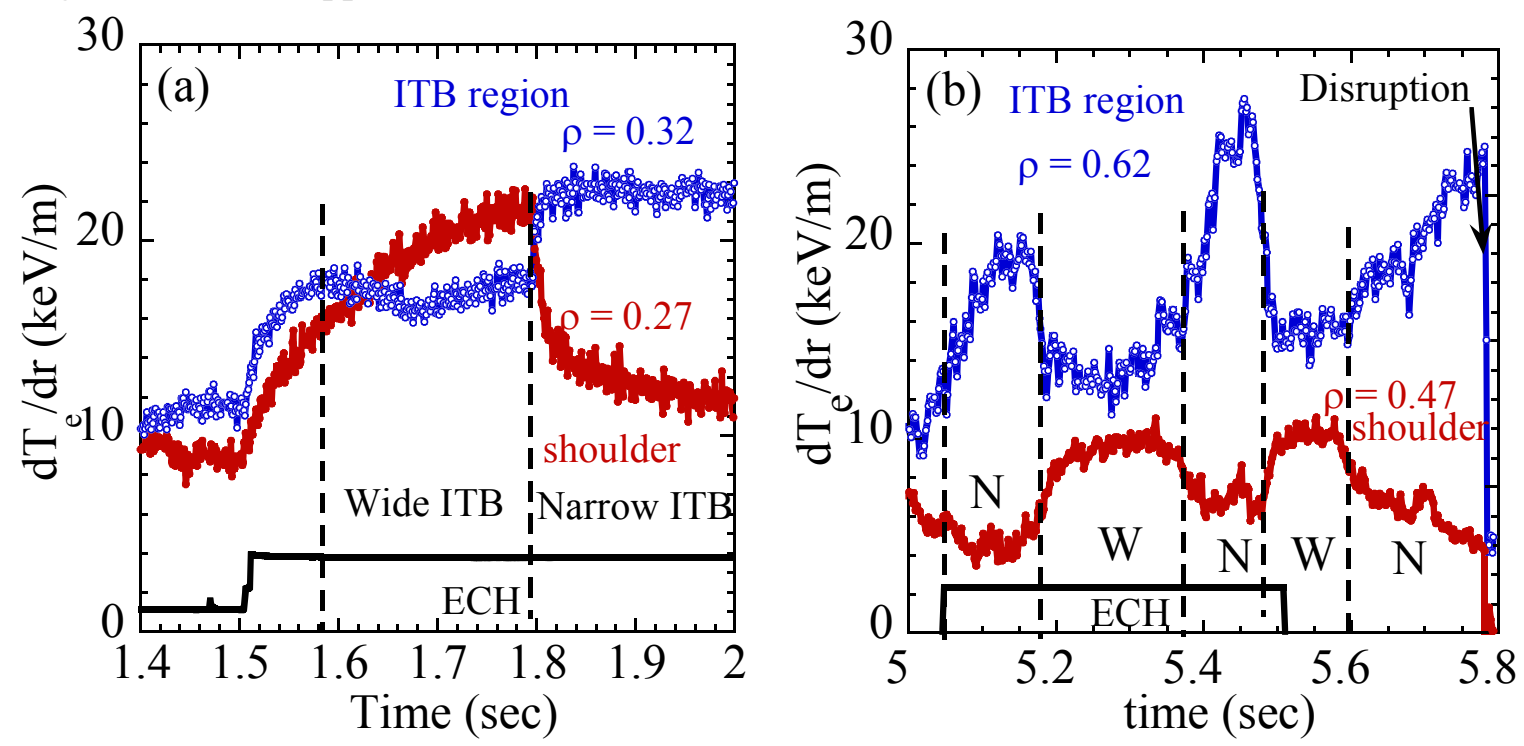

FIG.5 Time evolutions of the electron temperature gradients in the ITB region and at the ITB shoulder in (a) LHD and (b) JT-60U. The transition from a weak ITB to a strong, narrow ITB and the back-transition are observed in LHD and a spontaneous oscillation between weak, wide and strong, narrow ITBs is clearly observed in JT-60U. 
A spontaneous transition from a weak ITB to a strong, narrow ITB is observed during the phase of constant heating power. The thermal diffusivities are calculated at the location with the highest temperature gradient of the ITB region and at the location slightly inner side of the ITB region, where the transport significantly changes at the transition from wide ITB to narrow ITB. Figure 6 shows the temperature dependence of thermal diffusivity during constant heating power. Radial profiles of electron temperature are also plotted and the locations of interest are indicated with arrows. The $\chi_{\mathrm{e}}$ values at $\rho=0.27$ and 0.32 are almost identical in the early phase of the wide weak ITB in LHD. After the transition from a wide ITB to a narrow ITB, the $\chi_{\mathrm{e}}$ value at $\rho=0.32$ starts to drop while the $\chi_{\mathrm{e}}$ value at the inner part of the plasma $(\rho=0.27)$ increases sharply.

In JT-60U both the transition and back-transition between wide ITB and narrow ITB are observed. The $\chi_{\mathrm{e}}$ values are almost identical both at $\rho=0.45$ and 0.65 in the wide weak ITB phase. After the transition to a narrow ITB, the $\chi_{\mathrm{e}}$ at $\rho=0.45$ in the core region increases (the $T_{e}$ gradient decreases) associated with the decrease of $\chi_{\mathrm{e}}$ at $\rho=0.65$ at the highest gradient region. It should be noted that the narrowing of ITB region is associated with the improvement transport at the highest gradient region. The narrowing of ITB is not just a shrink of ITB regime but the more localization of improved confinement. Therefore the electron temperature inner region ( $\rho=0.27$ in LHD and $\rho=0.45$ in JT-60U) slightly increases even the thermal diffusivity becomes large by a factor of two during the narrowing of ITB. It is interesting that $\chi_{\mathrm{e}}$ values slightly separated in space have opposite temperature dependences during the transition and back-transition phases. This fact shows the strong interaction of turbulent transport in space, where turbulence suppression at certain locations of the plasma causes the enhancement of turbulence and thermal diffusivity nearby.
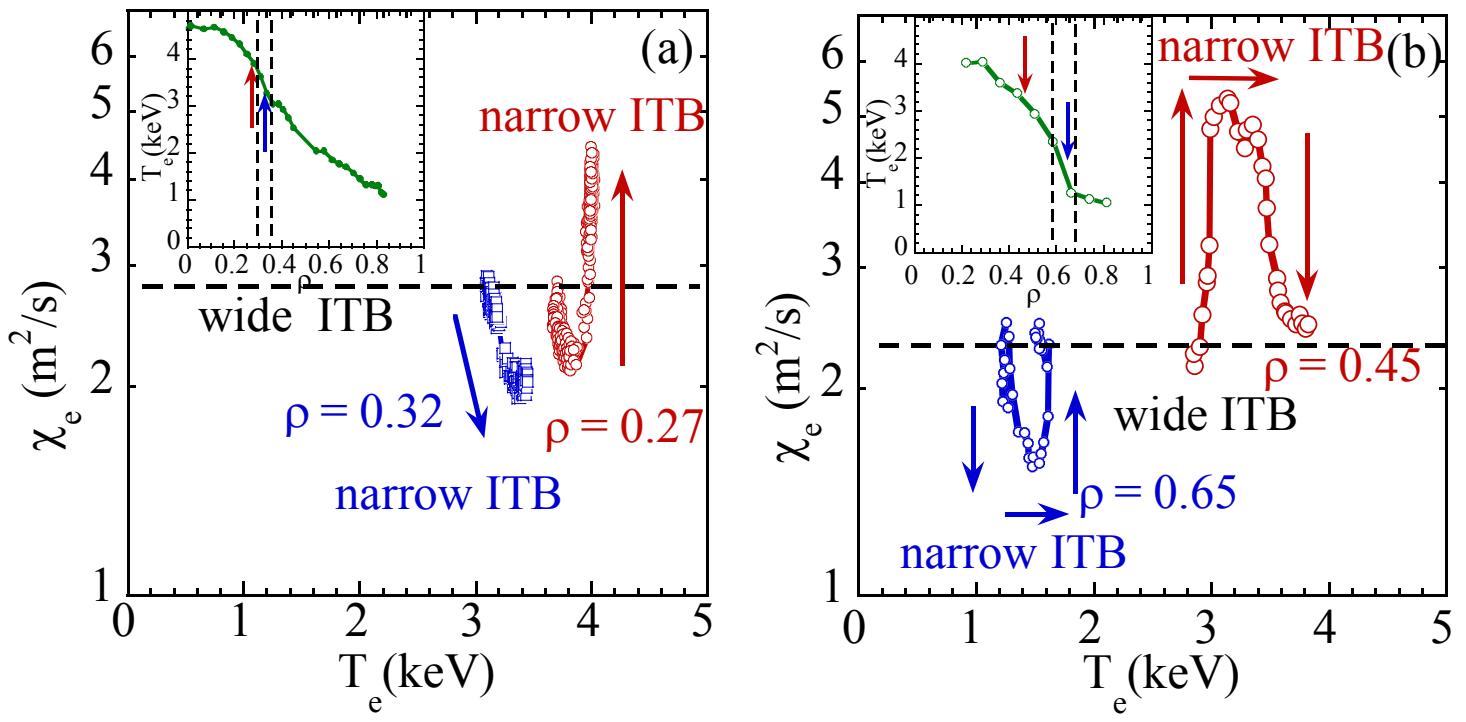

FIG.6 Thermal diffusivity as a function of $T_{e}$ at the highest gradient ITB region and at the shoulder in the narrow strong ITB in (a)LHD and (b)JT-60U. The spontaneous oscillation between weak wide and narrow strong ITB is observed in JT-60U. Radial profiles of electron temperature at the period of narrow ITB are also plotted. Arrows indicate the location where the thermal diffusivities are calculated with the transport analysis.

\section{Discussion and Summary}

Transport analysis during the transient phase of heating (a dynamic transport study) is applied to plasmas with internal transport barriers (ITBs) in the Large Helical Device (LHD) heliotron and in the JT-60U tokamak. In the dynamic transport study 1) a slow transition between two transport branches is observed, 2) the time of the transition from the L-mode plasma to the ITB plasma is clearly determined by the onset of flattening of the temperature 
profile in the core region for the first time and 3) a spontaneous phase transition from a weak, wide ITB to a strong, narrow ITB and back-transition are observed both in LHD and JT-60U.

The time scale of the slow transition between two transport branches is on the order of the energy confinement time. The time scale of the transition from the L-mode plasma to the ITB mode plasma is also on the order of the energy confinement time, which make it difficult to specify the starting time of the TB formation. In a strong ITB, the flattening of the temperature in the core region is often observed. This flattening starts in the early phase of the ITB formation not in the later time of the ITB when the electron temperature becomes high. Therefore the flattening is considered to be due to the localization of improved transport during the formation of the ITB. The flattening of the core region of the ITB transition and the back-transition between a wide ITB and a narrow ITB suggest the strong interaction of turbulent transport in space, where turbulence suppression at certain locations in the plasma causes the enhancement of turbulence and thermal diffusivity nearby. Because of this mechanism, the ITB is naturally localized in space and the location and width of the ITB can change by itself without a significant change in the magnetic structure. This behaviour also indicates that there are multi meta-stable states in the transport and the spontaneous transition between these meta-stable states occurs on a transport time scale.

\section{Acknowledgement}

The authors would like to thank to Dr.K.Itoh for useful discussion on the interpretation of the experimental data and the technical staff in LHD and JT-60U for their support of these experiments. This work is partly supported by a Grant-in-aid for Scientific research (15206106) and the Grant-in-Aid for Specially-Promoted Research (18206094) of MEXT Japan. This work is also partly supported by NIFS05LUBB510.

\section{References}

[1] ITOH, K., ITOH, S.-I., FUKUYAMA, A., : Transport and Structural Formation in Plasmas (IOP, England, 1999).

[2] HUBBARD, A.E., et al., Plasma Phys. Control. Fusion 44 A359 (2002).

[3] IDA, K., INAGAKI, S., SAKAMOTO, R., TANAKA, K., et al., Phys. Rev. Lett. 96 (2006) 125006.

[4] FUJITA, T., et. al., Phys. Rev. Lett. 78 (1997) 2377.

[5] WAGNER, F., et al., Phys. Rev. Lett. 49, (1982) 1408.

[6] GROEBNER, R.J., et al., Phys. Rev. Lett. 64, (1990) 3015.

[7] IDA, K., et. al., Phys. Rev. Lett. 65, (1990) 1364.

[8] FUJISAWA, A., IGUCHI, H., MINAMI, T., et al., Phys. Rev. Lett. 82, (1999) 2669.

[9] IDA, K., SHIMOZUMA, T., FUNABA, H., Phys. Rev. Lett. 91, (2003) 085003.

[10] SHIMOZUMA, T., KUBO, S., IDEI, H., et al., Phys. Plasma 45, (2003) 1183.

[11] TAKEIRI, Y., SHIMOZUMA, T., KUBO, S., et al., Phys. Plasmas 10, (2003) 1788

[12] IDA, K., et al., Phys plasmas 11 (2004) 2551

[13] KAMADA, Y., et al., Fusion Science and Technology 42 (002) 185.

[14] IDA, K., FUJITA, T., FUKUDA, T., SAKAMOTO, Y., et al., Plasma Phys Control Fusion 46 (2004) A45.

[15] SAKAMOT, Y., et al., Nucl. Fusion 44 (2004) 876.

[16] TAKENAGA, H., et. al., Plasma Phys. Control. Fusion 48 (2006) A401

[17] MIYAZAWA, J., et al., Plasma. Phys. Control. Fusion 47 (2005) 801.

[18] ITOH, S.-I. and ITOH, K., Plasma Phys. Control. Fusion 43 (2001) 1055.

[19] FUJITA, T., et al., Plasma Phys. Control. Fusion 46 (2004) A35

[20] NEUDATCHIN S.V. et al., Plasma Phys. Control. Fusion 44 (2002) A383.

[21] IDE, S., et. al., this conference EX/P1-5 